\title{
A unifying molecular mechanism underlying the association of CARD14 alleles with autoinflammatory and T-cell mediated skin disorders
}

\author{
D Berki ${ }^{1}$, S-E Choon², AD Burden ${ }^{3}$, C Griffiths ${ }^{4}$, C Smith ${ }^{1}$, J Barker ${ }^{1}$, F Capon ${ }^{\text {* }}$ \\ From 8th International Congress of Familial Mediterranean Fever and Systemic Autoinflammatory Diseases \\ Dresden, Germany. 30 September - 3 October 2015
}

\begin{abstract}
Introduction
The CARD14 (Caspase Recruitment Family Member 14) locus encodes a scaffold protein that mediates NF-kB signalling in keratinocytes and is therefore crucial to the maintenance of skin immune homeostasis. In keeping with this notion, gain-of-function CARD14 mutations have been observed in patients with plaque psoriasis and pityriasis rubra pilaris, two skin disorders mediated by abnormal $\mathrm{T}$ cell activation. More recently, a CARD14 missense variant has been tentatively associated with generalised pustular psoriasis (GPP), an auto-inflammatory condition characterised by acute episodes of skin pustulation and systemic upset.
\end{abstract}

\section{Objectives}

The aim of this study was to establish whether CARD14 alleles are genuinely associated with GPP and to investigate the molecular mechanism underlying any effect on disease risk.

\section{Patients and methods}

We investigated an extended case cohort $(n=100)$ ascertained in Europe and East Asia. As all disease alleles described to date cluster to exons 3 and 4, we screened this mutation hotspot in all patients. We also sequenced the entire CARD14 coding region in a subset of 16 individuals. We analysed population matched, control genotypes $(n=997)$ that were generated in-house or had been previously released by the 1000 Genomes Consortium. Finally, we investigated the accumulation of CARD14 oligomers by western blotting, following the transfection of HEK293 cells with wild-type or mutant cDNA constructs.

${ }^{1}$ King's College London, London, UK

Full list of author information is available at the end of the article

\section{Results}

We found that a non-conservative p.Asp176His substitution was significantly associated with GPP in the Chinese and Japanese populations (combined $P=0.0001$; OR:5.3). Bioinformatics showed that this change had pathogenic potential and was likely to disrupt the coiled coil of CARD14. Importantly, our analysis predicted a similar effect for p.Glu138Ala and p.Leu156Pro, two disease alleles previously associated with psoriasis and pityriasis rubra pilaris. Since the coiled coil domain of CARD14 mediates protein oligomerization, we investigated the effects of the above mutations on the accumulation of CARD14 aggregates. We found that all three disease alleles caused spontaneous protein oligomerization.

\section{Conclusion}

Given that CARD14 oligomerization is a pre-requisite for downstream signal transduction, our results indicate that disease alleles promote abnormal NF-kB signalling by causing constitutive protein aggregation. Thus, our work points to a unifying pathogenic mechanism underlying the effects of CARD14 mutations on auto-inflammatory and T-cell mediated disorders.

\section{Authors' details \\ ${ }^{1}$ King's College London, London, UK. ${ }^{2}$ Hospital Sultanah Aminah, Johor Bahru, Malaysia. ${ }^{3}$ University of Glasgow, Glasgow, UK. ${ }^{4}$ University of} Manchester, Manchester, UK.

Published: 28 September 2015

doi:10.1186/1546-0096-13-S1-050

Cite this article as: Berki et al:: A unifying molecular mechanism underlying the association of CARD14 alleles with autoinflammatory and Tcell mediated skin disorders. Pediatric Rheumatology 2015 13(Suppl 1):O50. 